Okajimas Folia Anat. Jpn., 81(2-3): 45-48, August, 2004

\title{
Branch of Mylohyoid and Lingual Nerves on Submandibular and Submental Triangles
}

By

\author{
Iwao SATO, Masataka SUNOHARA, Ryuji UENO and Shyunji YOSHIDA \\ Department of Anatomy, School of Dentistry at Tokyo, Nippon Dental University, Tokyo, 102-8159, Japan
}

- Received for Publication, June 7, $2004-$

Key Words: Gross anatomy, Mylohyoid nerve, Submandibular triangle, Submental triangle

\begin{abstract}
Summary: The mylohyoid nerve (MN) displays several branches in the posterior, intermediate, and anterior region of the mylohyoid muscle (MM) as it courses on the internal surface of the mandibular body. Branches in the intermediate region were found in $66 \%$ of the cases ( 272 out of 413 sides). In the submandibular triangle, one or two large branches of the MN communicated with the lingual nerve at submandibular triangle and submental triangle in $1.45 \%$ of the cases (6 out of 413 sides). These distributions of nerve supply are an important in the operations of radical neck dissection on the submandibular triangle.
\end{abstract}

The mandibular nerve divides into two branches, the lingual nerve (LN) and the inferior alveolar nerve (IAN), at the mandibular notch. The IAN descends to the mandibular nerve branches on the inner mylohyoid muscle (MM). Mandibular nerve has anastomotic branches connecting to the lingual nerve (LN) after leaving laterally from the foramen ovale (Clemente, 1985). Chorda tympani join the $\mathrm{LN}$ in the deep region behind the lateral pterygoid muscle. The IAN is also branched into the mylohyoid nerve $(\mathrm{MN})$ at the mandibular foramen. There are a few communications between the MN and the LN in the submental triangle. Kameda (1952) has reported the MN communicated with the LN in $46.3 \%$ ( 74 out of 160 sides) of the cases.

The communication rate of the $\mathrm{LN}$ and the MN in Kameda reports (1952) is high in compared to that of our data, however, this reports is no detailed in course, supply, and any region in two nerves. Radical neck dissection needs the detailed information of nerve supply in the submandibular triangle (Beahrs, 1977; Swift, 1970; Feldman and Applebaum, 1977). Trail and Lubritz (1974) also indicated complete removal of tumors on the submandibular gland required radical excision of tissue around them, including neck dissection. Therefore we observed nerve supply on submandibular triangle and submental triangle in detail.

\section{Materials and Methods}

We examined the mylohyoid nerve branches under a binocular microscopy, 413 sides of 272 mandibles from adult Japanese cadavers (161 males and 111 females; 141 both sides, 60 right side only and 71 left side only) for passage and supply. We also observed the communications between the mylohyoid nerve (MN) and the lingual nerve (LN) in the submandibular triangle and submental triangle. Using forceps, we removed the entire mucous membrane, the connective tissues of the mylohyoid muscle (MM), parts of the ramus of the submental arteries, and veins at submandibular triangle and submental triangle. In each case, the communications between the MN and the LN and their distribution were analyzed and photographed.

\section{Results}

We observed the course and the communications between the mylohyoid nerve (MN) and the lingual

* Correspondence to: Iwao Sato, PhD., 1-9-20 Fujimi Chiyoda-ku, Tokyo, Japan, 102-8159, Telephone fax, +81-3-5216-3752, E-mail: iwaoa1@tokyo.ndu.ac.jp 


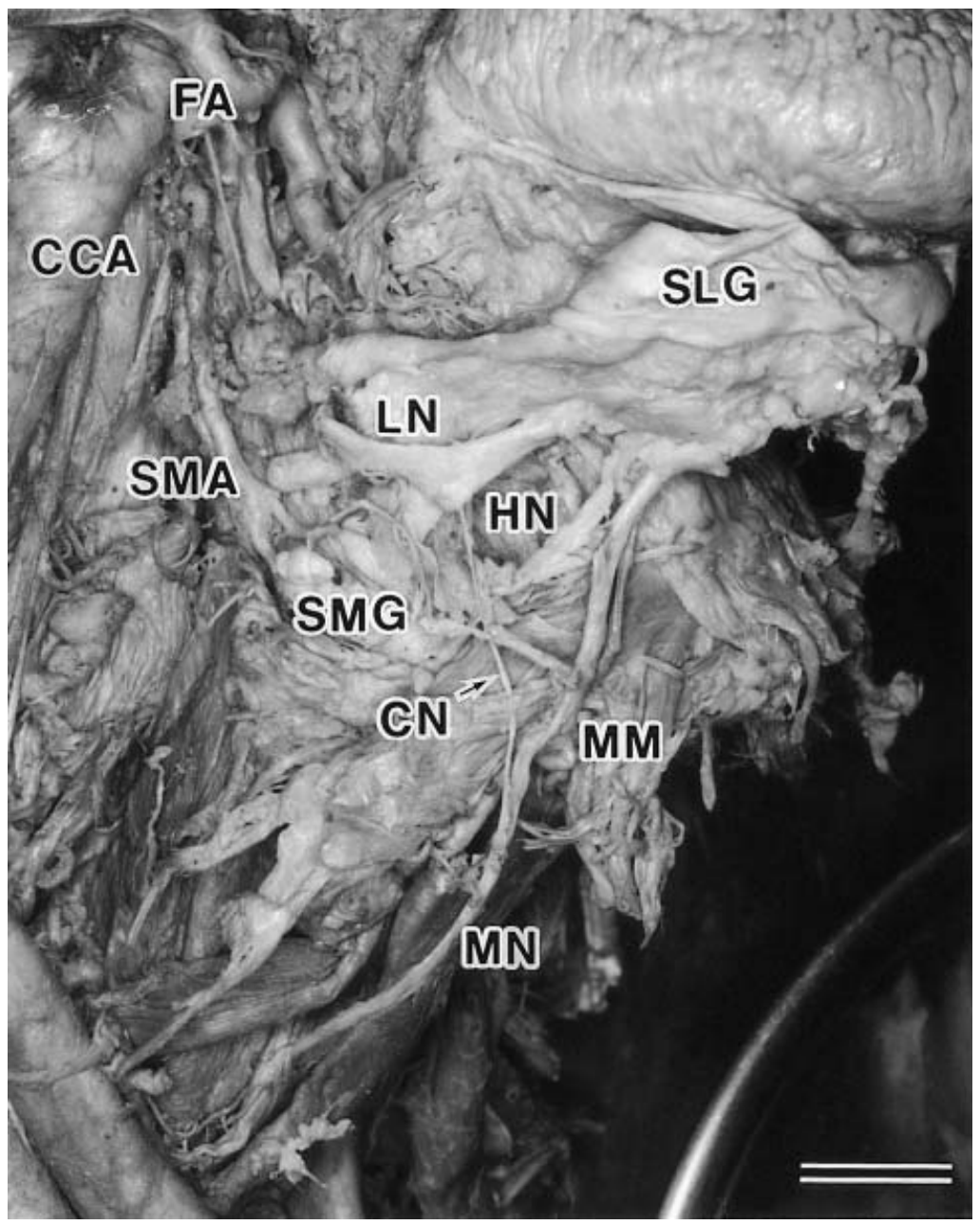

Fig. 1. Case 1 (right side, 83 years old, female).

A small branch $(\mathrm{CN}$, arrow) of the mylohyoid nerve $(\mathrm{MN})$ was connected with the lingual nerve (LN) at the posterior region of the MM. Bar $=2 \mathrm{~cm}, \mathrm{CN}$, communicating nerve; CCA, common carotid artery; FA, facial artery; HN, hypoglossal nerve; MM, mylohyoid muscle; SLG, sublingual gland; SMA, submental artery; SMG, submandibular gland.

nerve (LN) on submandibular triangle and submental triangle. No significant differences existed with respect to age, side, and sex.

\section{The branch of the $M N$}

The MN passes through the mylohyoid muscle (MM) and runs parallel to the submental artery and vein. There are several branches of $\mathrm{MN}$ in the posterior, the intermediate and the anterior regions of $\mathrm{MM}$ on the internal surface of the mandibular body. In the posterior region of the MM, branches with two or three small ramus innervated in the internal border of the MM were found in $44 \%$ of the sides examined ( 180 out of 413 sides). In the intermediate region that percentage was $66 \%$ (272 out of 413 sides), and in the anterior region, the percentage was $35 \%$ (144 out of 413 sides).
The branches between the MN and the LN in typical six cases

When the MN passed through the MM, one or two large branches of the MN connected with the $\mathrm{LN}$ on the submandibular triangle. These anastomotic branch terminals of the $\mathrm{MN}$ were found in $1.45 \%$ of cases ( 6 out of 413 sides).

Case 1 (right side, 83 years old, female): A small branch of the MN was connected with the $\mathrm{LN}$ in the posterior region of the MM. This small nerve coursed beneath the submental artery and vein, and spread into the mental triangle. Case 2 (left side, 60 years old, female): A large branch from trunk of the MN connected with the $\mathrm{LN}$ in the posterior region of the MM. The submental nerve coursed beneath the submental vein and artery. Case 3 (left side, 59 years old, male): Two branches of the MN 


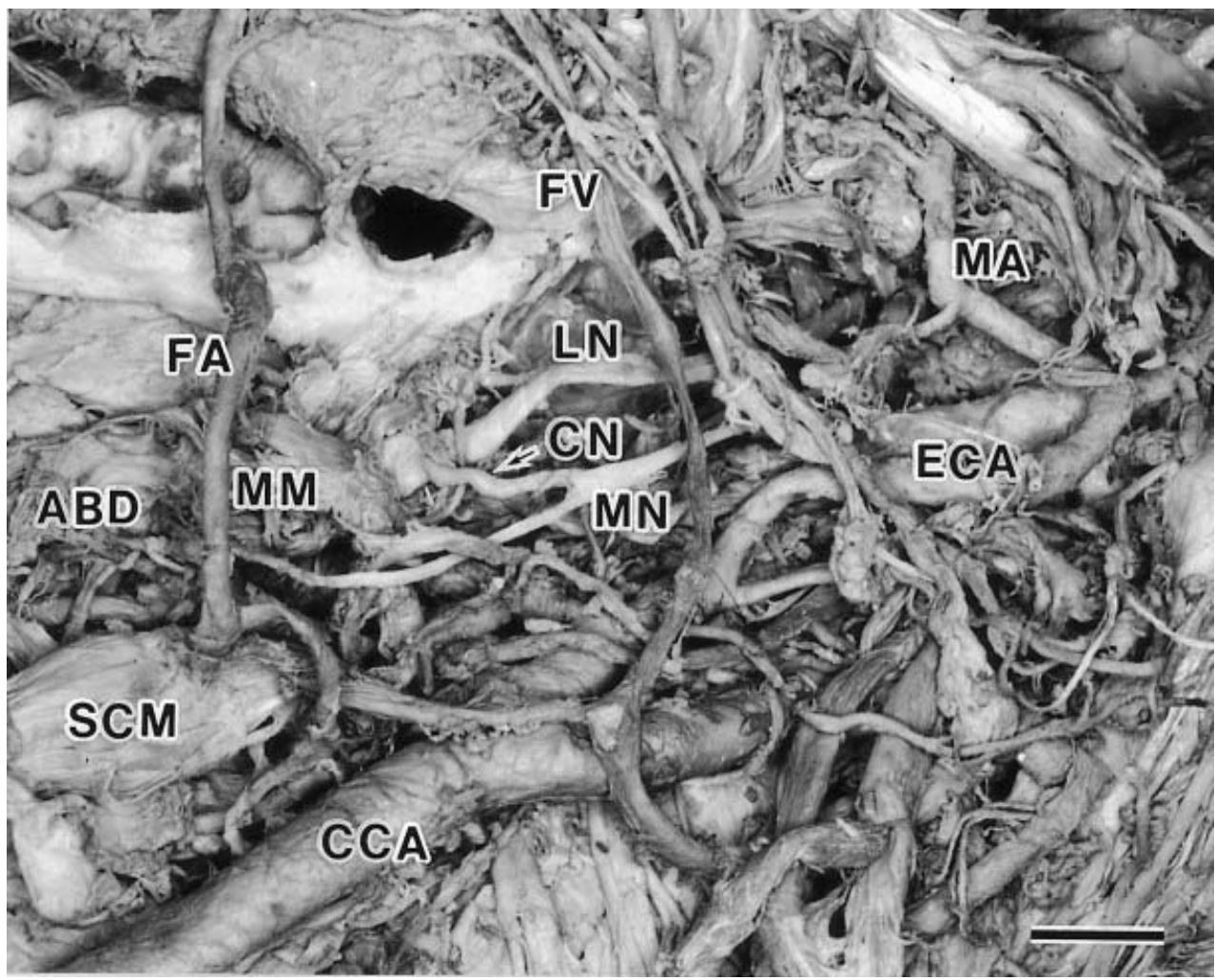

Fig. 2. Case 4 (left side, 60 years old, female).

A large branch $(\mathrm{CN}$, arrow) from trunks of the mylohyoid nerve $(\mathrm{MN})$ connected with the lingual nerve $(\mathrm{LN}) . \mathrm{Bar}=2 \mathrm{~cm}, \mathrm{CN}$, communicating nerve; CCA, common carotid artery; ECA, external carotid artery; FA, facial artery; FV, facial vein; MA, maxillary artery; MM, mylohyoid muscle; SCM, sternocleidomastoid muscle.

connected with the LN. One branch connected in the intermediate region of the $\mathrm{MM}$ and another larger branch connected in the posterior region of the MM. The submental nerve also divided into two branches and ran beneath the submental artery.

\section{Discussion}

In clinical and pathological studies, the metastasis of the cervical lymph node (Shah, 1990), the lymph node in the oral cavity (Feldman and Applebaum, 1977), and the base of tongue where the $\mathrm{LN}$ runs near the both lateral regions in the oropharynx (Byers, 1985) were reported. In the primary salivary gland, tumors on the submandibular and the sublingual glands were also reported (Trail and Lubritz, 1974). They reported an important display in the treatment of submental and submandibular triangles in neck dissections. From our observation of 413 sides of mandibules from Japanese adult cadavers, the mylohyoid nerve (MN) had several branches in the posterior, the intermediate and the anterior regions of the mylohyoid muscle (MM) on the internal surface of mandibular body. Most frequently, we observed the branch of the $\mathrm{MN}$ in the intermediate region $(66 \%$ of the cases). The submaxillary gland, which is downward to the mandible, the posterior belly of the digastric muscle, and the posterior region of the MM, filled the submaxillary triangle. The hypoglossal nerve and the LN are closely associated with the gland and its duct from the intermediate to the posterior regions of the MM. Therefore these regions are important landmarks for the neck dissection. Beahrs (1977) also suggested that the MM is an important landmark in radical neck dissection. When removing the anterior belly of the digastric muscle from the MM in the submental and the submandibular triangles, we need to pay extra attention to the $\mathrm{LN}$ and the hypoglossal nerve as they make complex arrangement in these triangles.

In $1.45 \%$ of cases ( 6 of 413 sides), one or two large branches of the MN connected with the LN in submandibular triangle and submental triangle. This percentage is significantly different from that reported by Kameda (1952) which is $46.3 \%$ (74 out of 160 sides) in the submental triangle. In the 


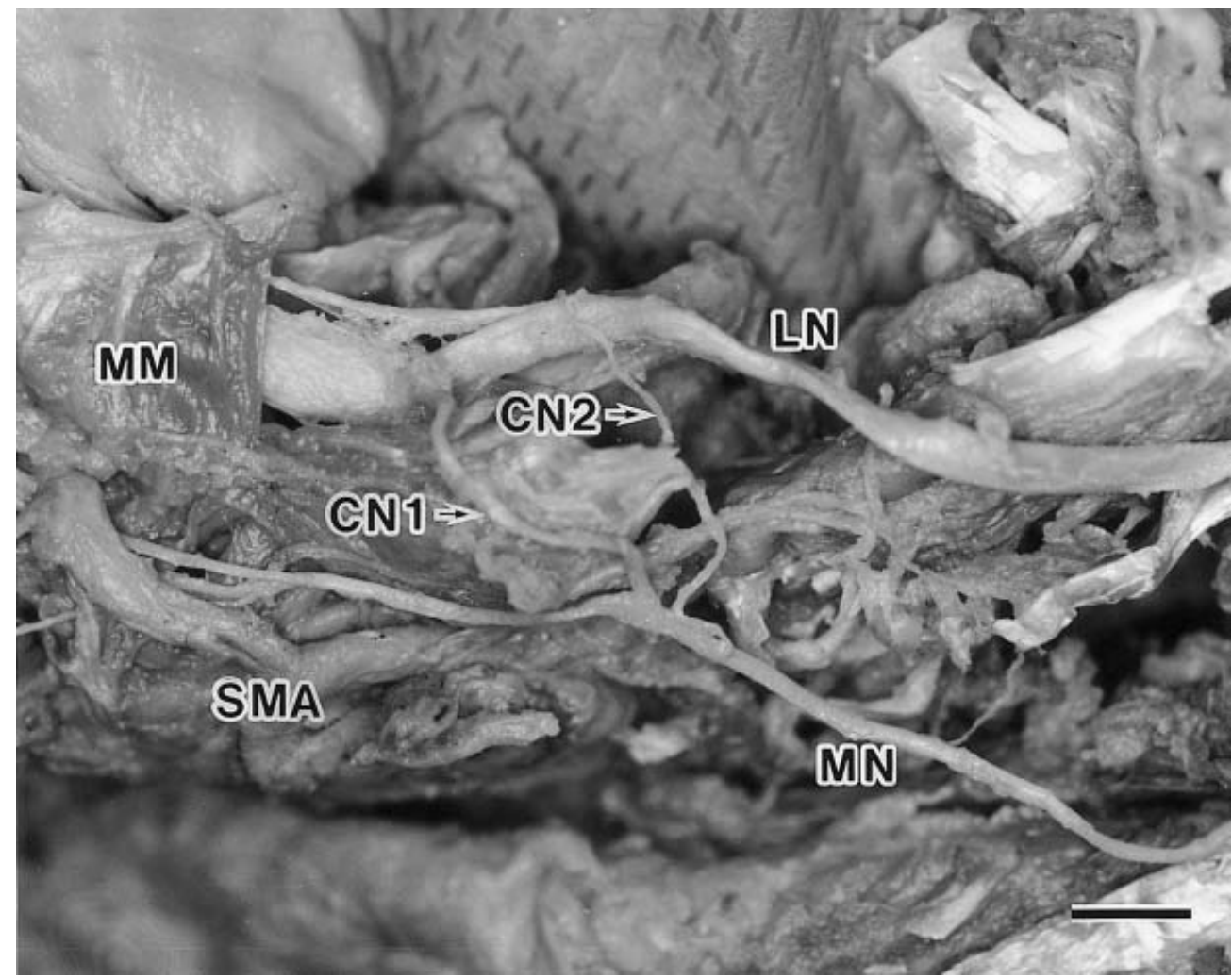

Fig. 3. Case 3 (left side, 59 years old, male).

One branches (CN2, arrow) connected at intermediate and other large branches (CN1, arrow) from trunks of the MN. $\mathrm{Bar}=1 \mathrm{~cm}, \mathrm{ABD}$, anterior belly of digastric muscle; $\mathrm{CN}$, communicating nerve; MM, mylohyoid muscle; SMA, submental artery.

superior region and the submental region of the mandible, most of the $\mathrm{LN}$ and the inferior alveolar nerve (IAN) connected with the branches of the facial nerve. They also connected with the hypoglossal nerve in anterior region of the hyoglossus muscle. There were many fine twigs of these nerves supplied in the region. Therefore, it is difficult to distinguish at a macroscopic level, the numerous branches of the LN and the IAN clearly in the deep submental and the submandibular triangles.

\section{Acknowledgements}

We wish to thank Prof. Noboru Goto (Chairman of Department of Anatomy, Showa University School of Medicine) and Prof. Susumu Kato (Department of Anatomy, The Jikei University School of Medicine) for their help with this research.

\section{References}

1) Beahrs OH. Surgical anatomy and technique of radical neck dissection. Surg Clin North Am 1977; 57:663-700.

2) Byers R. Modified neck dissection. A study of 967 cases from 1970 to 1980. Am J Surg 1985; 150:414-421.

3) Clemente CD. Gray's Anatomy of the Human Body, 30th ed. Clemente CD (Eds): pp 1168-1169, Williams \& Wilkins A Div of Waverly Press, Inc, Philadelphia, 1985.

4) Feldman DE and Applebaum EL. The submandibular triangle in radical neck dissection. Arch Otolaryngol 1977; 103:705-706.

5) Kameda K. Über den N. Mandibularis bei Japanern. Acta Anatomica Niigata'ensia Sectionis Anatomicae Universitais Niigata'ensis 1952; 28:1-24.

6) Shah JP. Patterns of cervical lymph node metastasis from squamous carcinomas of the upper aerodigestive tract. Am J Surg 1990; 160:405-409.

7) Swift TR. Involvement of peripheral nerves in radical neck dissection. Am J Surg 1970; 119:694-698.

8) Trail ML and Lubritz J. Tumors of the submandibular gland. Laryngoscope 1974; 84:1225-1232. 